\title{
EDITORIAL
}

\section{Why basic microbiology still matters}

\author{
Microbiology has been a foundation of our investigation into the basic principles of life, \\ but much remains to be discovered. Therefore, the focus on basic microbiology research \\ cannot be lost.
}

Through the study of microorganisms, the field of microbiology has made fundamental contributions to molecular biology. Indeed, the list of discoveries made through the investigation of microorganisms is long and includes the most central concepts of molecular biology. For example, a recent meeting at the Institut Pasteur in Paris, France, celebrated "the operon model and its impact on modern molecular biology" to mark the fiftieth anniversary of the publication of François Jacob and Jacques Monod's article describing this model of the regulation of gene expression. Before the operon model, Oswald Avery, Colin MacLeod and Maclyn McCarty (aided by a pancreatic extract) used Streptococcus pneumoniae to show that DNA is the 'transforming material', a finding that was later corroborated by the experiments of Alfred Hershey and Martha Chase, who showed that DNA is the heritable material in phages. The Avery, MacLeod and McCarty experiment was itself the extension of the famous experiments performed by Frederick Griffiths, which showed that bacteria have the ability to 'transform' - that is, to acquire traits from other strains during a co-infection. The genetic code was deciphered in large part by using phage mutants in bacteria, whereas the break from the central dogma, the formation of DNA from RNA by reverse transcriptase, was discovered in viruses.

However, the focus within microbiology has been changing from basic microbiology to translational and applied microbiology over the years. Many researchers still investigate very basic aspects of microbiology — including such fundamental questions as how bacteria and archaea divide; how viruses enter host cells, replicate and form infectious virions; and how fungi grow and develop their multiple forms - but there is increasing pressure to investigate topics that are 'translational' or that are tied directly to human health. Indeed, in many cases, microbiology departments are reluctant to hire a microbiologist who does not study bacterial pathogenicity or applied microbiology. This is understandable, to some degree, as people are naturally curious about the what makes them ill and indeed many interesting discoveries have resulted from this line of research (although it can be argued that pathogenicity is a growth adaption to a specific environment: the eukaryotic host).
Nonetheless, there are many reasons why the study of basic microbiology remains important. First and foremost, many fundamental questions remain to be answered. Even the bacterial cell wall, which has been the focus of investigation for $>50$ years, still has secrets to reveal, as indicated by the recent discoveries of the lipoteichoic acid biosynthesis pathway in Gram-positive bacteria and the links between the outer membrane and peptidoglycan synthesis in Gram-negative bacteria. Furthermore, bacteria, archaea, phages, protists and viruses play a fundamental part in the turnover of key nutrients in the environment, as drivers of the carbon and nitrogen cycles in the oceans and the soil, for example. Further investigation of these processes will allow us to understand them better, and this will shed light on the important role that these organisms may have in climate change. Closer to home, insights into the microbiota associated with our bodies have revealed the presence of many poorly characterized, but often abundant, genes and bacteria.

The functions of many genes in the genomes of even the best studied organisms, such as Escherichia coli and Bacillus subtilis, remain unknown. Often these genes do not resemble other, characterized, genes in the databases, allowing for the possibility that interesting new pathways remain to be discovered. Viruses and phages are even more likely to carry genes that have no similarity to anything in the databases. Finally, our best chance to understand the basics of life is through the study of microorganisms, as they are the smallest, simplest freeliving organisms. They are nestled firmly at the base of the tree of life, and if we want to understand how life works at the molecular level, it is crucial to continue and expand basic microbiology research.

The study of microorganisms has yielded tremendous insight into the basic principles of life but, despite all that we have learned, we are far from understanding in detail how these organisms function. The relative (but sometimes deceptive) simplicity of microorganisms makes them ideally suited for many fundamental discoveries, and many such discoveries are probably yet to come, if microbiology, and specifically basic microbiology, remains a well-funded discipline. 